\title{
Some Exact Solutions of Plane Steady Hydromagnetic Flow with Variable Viscosity
}

\author{
Pankaj Mishra (Corresponding author)
}

Department of Mathematics, Faculty of Science

Banaras Hindu University Varanasi-221005, U. P., India

Tel: 91-945-158-1466 E-mail: pankaj1mishra@yahoo.co.in

R. B. Mishra

Department of Mathematics, Faculty of Science

Banaras Hindu University Varanasi-221005, U. P., India

Tel: 91-941-538-9701_E-mail: rbmishra_bhu@yahoomail.com

Atul K. Srivastava

Department of Mathematics, Faculty of Science

Banaras Hindu University Varanasi-221005, U. P., India

Tel: 91-945-421-1945Ｅ-mail: atulshaswat@gmail.com

First author is highly thankful to Council of Scientific and Industrial Research, Govt. of India, New Delhi-110012, for providing financial support to carry out the present research work.

\section{Abstract}

The aim of the present paper is to find some exact solutions of steady two dimensional finitely conducting incompressible fluid flow under the presence of transverse magnetic field using transformation of variables. We have considered, vorticity distribution proportional to the stream function perturbed by a quadratic stream. The results are shown by various graphs.

Keywords: Steady flow, Navier stokes equations, Exact solution, Variable viscosity

\section{Nomenclature}

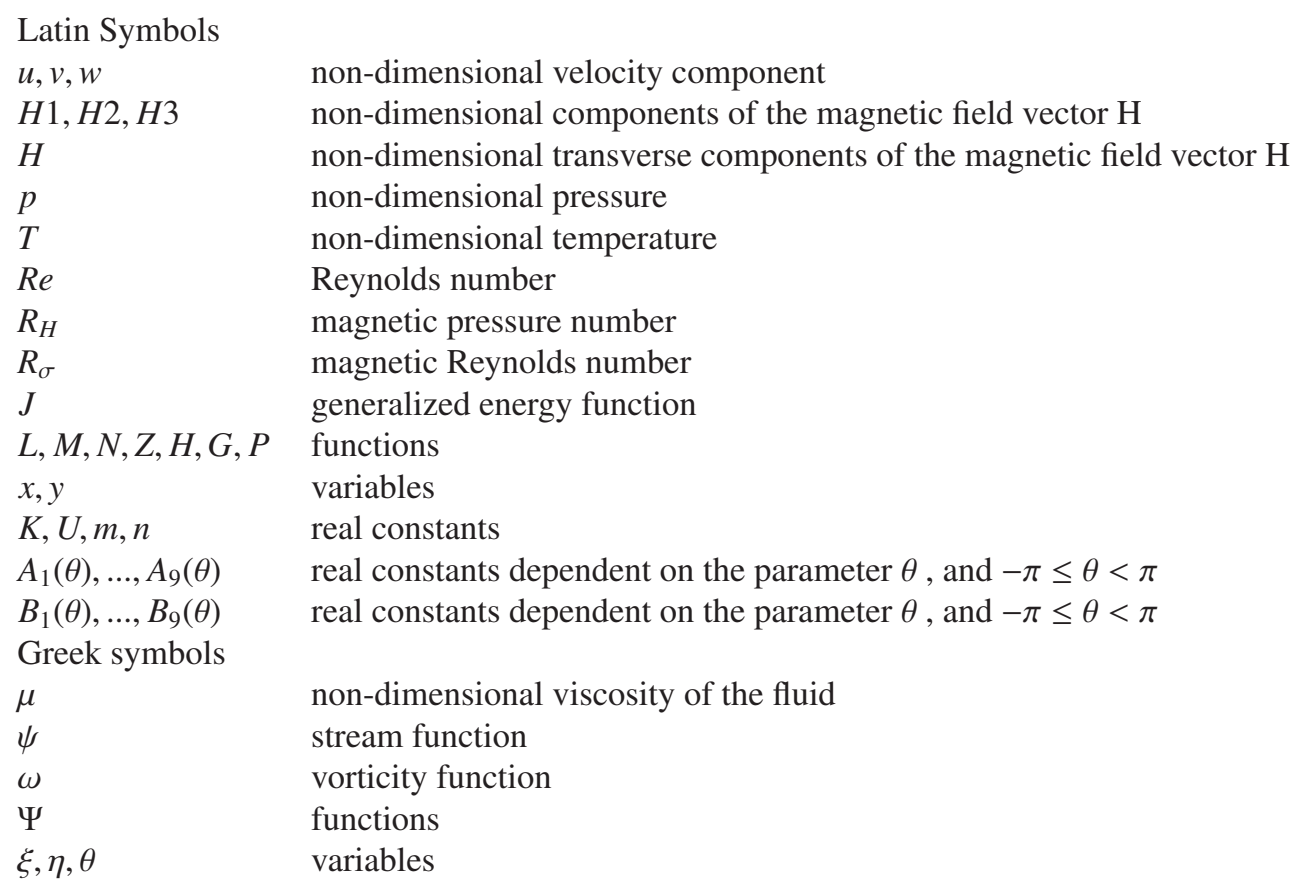

\section{Subscripts}


$x, y, z, x x, y y$ differentiation with respect to Cartesian coordinates $\mathrm{x}$ and $\mathrm{y}$.

$\xi, \eta, \xi \xi, \eta \eta \quad$ differentiation with respect to $\xi$ and $\eta$.

superscripts

dimensional quantities

\section{Introduction}

In order to describe the flow behavior of any fluid, we have to solve the Navier-Stokes equations arising in fluid flow. The importance of Navier-Stokes equations comes from their wide applicability for different kind of fluid flow, ranging from thin film to large scale atmospheric, even cosmic flows. However, Navier- Stokes equations are highly non-linear in nature and hence we face difficulties in solving them exactly. The full set of general solution of Navier-Stokes equations has not been found and is an open problem till the date. In order to overcome this difficulty one adopt transformations, inverse or semi- inverse method for the reformulation of equations in solvable form. Following the Martin's formulation (Martin, M.H., 1971), some researchers (Chandna, O.P., et al, 1982; Siddiqui, A.M., et al, 2008) have used hodograph transformation (Ames, W.F., et al, 1965) in order to linearized the system of governing equations and successfully got some exact solutions. Some authors (Chandna, O.P., et al, 1994; Hayat, T., et al, 1988 ) have used inverse method (Nemenyi, 1951) where some a priori condition is assumed about the flow variables and have found some exact solutions.

The above said solutions have been found for the flow of fluid with constant viscosity. But in many situations in the fluid flow, where the pressure and temperature gradients are high or in case of electrically conducting fluid flow where the magnetic field plays dominant role, the viscosity is no longer constant (Myers, et al, 2006; Sunil, et al, 2008; Kannan, et $a l, 2008)$. So In order to study fluid flows in such situations, we have to consider the viscosity of fluid as a variable, which gives rise the corresponding Navier-Stokes equations in more complicated form. The exact solution of Navier-Stokes equations for the fluid of variable viscosity are rare and very few work has been done in this aspect. As for the analytical solution is concern, Martin's approach, where system of equations are reformulated in curvilinear coordinates, was previously employed by Naeem in the compressible fluid of constant viscosity. This work was extended by Naeem and Nadeem (1996) for incompressible fluid of variable viscosity. Naeem (1994), utilizing one parameter group of transformation, transformed the governing equations of an incompressible fluid with variable viscosity into a system of ordinary differential equation and successfully got some exact solutions.

Moreover, exact solutions of the steady plane incompressible fluid flow with variable viscosity, employing transformation of variables (Naeem, et al, 2009) and von-mises variables (Naeem, et al, 2001) have been obtained. Recently Naeem and Jamil (2006), defined a one dimensional transformed variable $\xi=(x \cos \theta+y \sin \theta) ;-\pi \leq \theta \leq \pi$, converted the governing equations into simple ordinary differential equations and have got a class of exact solutions to flow of fluid of variable viscosity for which the vorticity function is proportional to the stream function perturbed by a uniform stream parrellel to X-axis. Further, Jamil and Khan (2006), using the same technique, extended this work by taking electrically conducting fluid of variable viscosity under the presence of transverse magnetic field and considering the vorticity distribution proportional to the stream function perturbed by a uniform stream, $U(x+y)$; where $U$ is a real constant, inclined to the $\mathrm{X}$-axis.

In the present analysis we have extended the work of Jamil and Khan (2006) to find some exact solutions of governing equations of the flow of electrically conducting fluid of variable viscosity under the presence of transverse magnetic field $\mathbf{H}=(0,0, H)$ by considering the vorticity distribution proportional to the stream function perturbed by a generalized quadratic stream, $U(x+y)-\left(A(\theta) x^{2}+B(\theta) y^{2}\right)$, where $A(\theta), B(\theta)$ are parametric constants depending on the parameter $\theta$.

\section{Equations of Motion}

The non-dimensional equation of steady plane flow of an incompressible electrically conducting fluid of variable viscosity under the presence of transverse magnetic field following Jamil and Khan (2006) are

$$
\begin{gathered}
u_{x}+v_{y}+w_{z}=0 \\
{\left[u u_{x}+v u_{y}+w u_{z}\right]=-P_{x}+\frac{1}{R_{e}}\left[\left(2 \mu u_{x}\right)_{x}+\left(\mu\left(u_{y}+v_{x}\right)\right)_{y}+\left(\mu\left(w_{x}+u_{z}\right)\right)_{z}\right]} \\
+R_{H}\left[H_{3}\left(H_{1 z}-H_{3 x}\right)-H_{2}\left(H_{2 x}-H_{1 y}\right)\right] \\
{\left[u v_{x}+v v_{y}+w v_{z}\right]=-P_{y}+\frac{1}{R_{e}}\left[\left(2 \mu v_{y}\right)_{y}+\left(\mu\left(v_{z}+w_{y}\right)\right)_{z}+\left(\mu\left(u_{y}+v_{x}\right)\right)_{x}\right]} \\
+R_{H}\left[H_{1}\left(H_{2 x}-H_{1 y}\right)-H_{3}\left(H_{3 y}-H_{2 z}\right)\right]
\end{gathered}
$$




$$
\begin{gathered}
{\left[u w_{x}+v w_{y}+w w_{z}\right]=-P_{y}+\frac{1}{R_{e}}\left[\left(2 \mu w_{z}\right)_{z}+\left(\mu\left(w_{x}+u_{z}\right)\right)_{x}+\left(\mu\left(v_{z}+w_{y}\right)\right)_{y}\right]} \\
+R_{H}\left[H_{2}\left(H_{3 y}-H_{2 z}\right)-H_{1}\left(H_{1 z}-H_{3 x}\right)\right], \\
\left(v H_{1}-u H_{2}\right)_{x}-\left(u H_{3}-w H_{1}\right)_{z}=\frac{1}{R_{\sigma}}\left(H_{1 x x}+H_{1 y y}+H_{1 z z}\right) \\
\left(w H_{2}-v H_{3}\right)_{z}-\left(v H_{1}-u H_{2}\right)_{x}=\frac{1}{R_{\sigma}}\left(H_{2 x x}+H_{2 y y}+H_{2 z z}\right) \\
\left(u H_{3}-w H_{1}\right)_{x}-\left(w H_{2}-v H_{3}\right)_{y}=\frac{1}{R_{\sigma}}\left(H_{3 x x}+H_{3 y y}+H_{3 z z}\right) .
\end{gathered}
$$

Since we have considered transverse plane flow i.e magnetic field is applied normal to the plane of flow, so we must have

$$
\begin{gathered}
(u, v, w)=(u, v, 0) \\
\left(H_{1}, H_{2}, H_{3}\right)=(0,0, H)
\end{gathered}
$$

Now in view of equation (8) we have the two dimensional form of governing equations as

$$
\begin{gathered}
u_{x}+v_{y}=0, \\
{\left[u u_{x}+v u_{y}\right]=-P_{x}+\frac{1}{R_{e}}\left[\left(2 \mu u_{x}\right)_{x}+\left(\mu\left(u_{y}+v_{x}\right)\right)_{y}\right],} \\
{\left[u v_{x}+v v_{y}\right]=-P_{y}+\frac{1}{R_{e}}\left[\left(2 \mu v_{y}\right)_{y}+\left(\mu\left(u_{y}+v_{x}\right)\right)_{x}\right]}
\end{gathered}
$$

with magnetic diffusion equation as

$$
u H_{x}+v H_{y}=\frac{1}{R_{\sigma}}\left[H_{x x}+H_{y y}\right]
$$

Where $H$ is the transverse component of magnetic field and $P=p+R_{H} \frac{H^{2}}{2}$. Further for non-dimensionalisation we have used the scaling parameters $L, U_{0}, \mu_{0}$ and $\rho U_{0}^{2}$ as reference length, velocity, viscosity and pressure. These are defined as

$$
u=\frac{u^{\prime}}{U_{0}}, v=\frac{v^{\prime}}{U_{0}}, w=\frac{w^{\prime}}{U_{0}}, \mu=\frac{\mu^{\prime}}{\mu_{0}}, p=\frac{p^{\prime}}{\rho U_{0}^{2}}, R_{e}=\frac{\rho U_{0} L}{\mu_{0}} .
$$

Symbols in the above equations have there usual meaning and are listed in the Nomenclature. Equation (9) implies the existence of stream function $\psi$ as

$$
u=\psi_{y}, \quad v=-\psi_{x} .
$$

Utilizing (14) in above equations we get

$$
\begin{gathered}
\psi_{x} \omega=-J_{x}+\frac{1}{R_{e}}\left[\mu\left(\psi_{y y}-\psi_{x x}\right)\right]_{y}, \\
\psi_{y} \omega=-J_{y}-\frac{4}{R_{e}}\left(\mu \psi_{x y}\right)_{y}+\frac{1}{R_{e}}\left[\mu\left(\psi_{y y}-\psi_{x x}\right)\right]_{x}, \\
\psi_{y} H_{x}-\psi_{x} H_{y}=\frac{1}{R_{e}}\left(H_{x x}+H_{y y}\right), \\
\omega=-\left(\psi_{x x}+\psi_{y y}\right), \quad J=P+\frac{1}{2}\left(\psi_{x}^{2}+\psi_{y}^{2}\right)-\frac{2 \mu \psi_{x y}}{R_{e}} .
\end{gathered}
$$

Now we let

$$
\psi_{x x}+\psi_{y y}=K\left(\psi-U x-U y+A x^{2}+B y^{2}\right) .
$$

Again from equation (18) and (19) we have

$$
\omega=-K \Psi
$$


where

$$
\Psi=\psi-U x-U y+A x^{2}+B y^{2} .
$$

Now using (20) and (21), equations (15) and (16) becomes

$$
\begin{gathered}
L_{x}=U K \Psi-2 K A x \Psi+\frac{1}{R_{e}}\left[\mu\left\{\left(\Psi_{y y}-\Psi_{x x}\right)+2(A-B)\right\}\right]_{y} \\
L_{y}=U K \Psi-2 K B y \Psi-\frac{4}{R_{e}}\left(\Psi_{x y}\right)_{y}+\frac{1}{R_{e}}\left[\mu\left\{\left(\Psi_{y y}-\Psi_{x x}\right)+2(A-B)\right\}\right]_{y}
\end{gathered}
$$

where $L=J-\frac{1}{2} K \Psi^{2}$, now using the Integrability criteria $L_{x y}=L_{y x}$ we get

$$
\left(M_{x x}-M_{y y}\right)-\frac{4}{R_{e}}\left(\mu \Psi_{x y}\right)_{x y}-2 K\left(B y \Psi_{x}-A x \Psi_{y}\right)+K U\left(\Psi_{x}-\Psi_{x}\right)=0
$$

where $M=\frac{\mu\left(\Psi_{y y}-\Psi_{x x}+2(A-B)\right)}{R_{e}}$.

(17) employing equation (21) becomes

$$
\left(\Psi_{y}+U-2 B y\right) H_{x}-\left(\Psi_{x}+U-2 A x\right) H_{y}=\frac{1}{R_{e}}\left(H_{x x}+H_{y y}\right)
$$

\section{Solutions}

In this section we find exact solutions of the governing equations. Using equations (19) and (21) we have

$$
\nabla^{2} \Psi=K \Psi+2(A+B)
$$

Now we seek the solution of the above equation of the form

$$
\Psi(x, y)=N(\xi)
$$

where

$$
\xi=(x \cos \theta+y \sin \theta), \quad-\pi \leq \theta \leq \pi .
$$

Using (27) in equation (26) we have

$$
N^{\prime \prime}(\xi)-K N(\xi)=2(A+B)
$$

Now for the solution of above equation we have two cases

Case (I): $K=-n^{2}, n \geq 0$

Case (II): $K=m^{2}, m \geq 0$

Now considering the case I we have the solution of (29) as

$$
N(\xi)=A_{1}(\theta) \cos \left(n \xi+A_{2}(\theta)\right)+\frac{2(A+B)}{n^{2}}
$$

Now using (27), (28) and (30) in (34) with suitable choice of

$$
A=-V \cot \theta, \quad B=V \tan \theta
$$

where

$$
\theta \in(-\pi, \pi) \backslash\left\{-\frac{\pi}{2}, \frac{\pi}{2}\right\}
$$

and $\mathrm{V}$ is some real constant, we have

$$
\begin{aligned}
& {\left[\left\{\frac{n^{2} A_{1}(\theta)}{R_{e}} \cos \left(n \xi+A_{2}(\theta)\right)-\frac{4 V}{R_{e}} \cot 2 \theta\right\} \mu\right]_{\xi \xi}} \\
& =n K A_{1}(\theta)[U(\cos \theta-\sin \theta)-2 V \xi] \sin \left(n \xi+A_{2}(\theta)\right)
\end{aligned}
$$

Thus using (28), (30) and (31) we the function $\Psi(x, y)$ as 


$$
\Psi(x, y)=A_{1}(\theta) \cos \left(n(x \cos \theta+y \sin \theta)+A_{2}(\theta)\right)-\frac{4 V}{n^{2}} \cot 2 \theta
$$

again using (21) and (33) we have the stream function as under:

$$
\begin{aligned}
\psi(x, y) & =A_{1}(\theta) \cos \left(n(x \cos \theta+y \sin \theta)+A_{2}(\theta)\right)-\frac{4 V}{n^{2}} \cot 2 \theta \\
& +U(x+y)+V\left(x^{2} \cot \theta-y^{2} \tan \theta\right)
\end{aligned}
$$

Now introducing new variable

$$
\eta=n \xi+A_{2}(\theta)
$$

the equation (32) becomes

$$
Z_{\eta \eta}=\left(A_{3}(\theta)+A_{0} \eta\right) \sin \eta
$$

where

$$
Z=\left(\cos \eta+A_{4}(\theta)\right) \mu
$$

$A_{0}=-\frac{2 K V R_{e}}{n^{4}}, A_{3}(\theta)=\frac{K R_{e}}{n^{3}}\left\{U(\cos \theta-\sin \theta)+\frac{2 A_{2}(\theta) V}{n}\right\}$ and $A_{4}(\theta)=-\frac{4 V \cot 2 \theta}{n^{2} A_{1}(\theta)}$.

Now on solving (36) and using (37) we get viscosity as

$$
\mu=-\frac{1}{\left(\cos \eta+A_{4}(\theta)\right)}\left[\left\{\left(A_{3}(\theta)+A_{0} \eta\right) \sin \eta+2 A_{0} \cos \eta\right\}+A_{5}(\theta) \eta+A_{6}(\theta)\right]
$$

where $A_{5}(\theta)$ and $A_{6}(\theta)$ are parametric constants. Using (17), (21), (28) and (35) we have

$$
H_{\eta \eta}=\left(A_{7}(\theta)+H_{0} \eta\right) H_{\eta}
$$

where $A_{7}(\theta)=\frac{R_{\sigma} U(\cos \theta-\sin \theta)}{n}$ and $H_{0}=-2 \frac{R_{\sigma}}{n}$. which gives the solution as

$$
H=A_{8}(\theta) \int e^{\frac{1}{2 H_{0}}\left(\left(A_{7}(\theta)+H_{0} \eta\right)^{2}\right.} d \eta+A_{9}(\theta)
$$

where $A_{8}(\theta)$ and $A_{9}(\theta)$ are parametric constants. Finally using (28) and (35) we have viscosity and magnetic field in cartisian coordinates.

Case (II): $K=m^{2}, m \geq 0$

Considering this case, solving equation (29) and then using (27), we have the solution for stream function as

$$
\Psi(x, y)=B_{1}(\theta) e^{m \xi}+B_{2}(\theta) e^{-m \xi}+\frac{2}{m^{2}}(A+B)
$$

Now using (27) (28) and (41) in (24) with suitable choice of

$$
A=-V \cot \theta, \quad B=V \tan \theta
$$

where

$$
\theta \in(-\pi, \pi) \backslash\left\{-\frac{\pi}{2}, \frac{\pi}{2}\right\}
$$

We have

$$
S_{\xi \xi}=\left\{B_{3}(\theta) e^{m \xi}+B_{4}(\theta) e^{-m \xi}\right\} \xi+B_{5}(\theta) e^{m \xi}+B_{6}(\theta) e^{-m \xi}
$$

where

$$
S=\mu\left\{\left(B_{1}(\theta) e^{m \xi}+B_{2}(\theta) e^{-m \xi}\right)+\frac{4 V}{m^{2}} \cot 2 \theta\right\}
$$

and

$$
\begin{aligned}
& B_{3}(\theta)=-\frac{2 K V R_{e} B_{1}(\theta)}{m}, \quad B_{4}(\theta)=\frac{2 K V R_{e} B_{2}(\theta)}{m} \\
& B_{5}(\theta)=\frac{B_{1}(\theta) R_{e} K U(\cos \theta-\sin \theta)}{m^{3}}, \quad B_{6}(\theta)=-\frac{B_{2}(\theta) R_{e} K U(\cos \theta-\sin \theta)}{m^{3}}
\end{aligned}
$$


using (39) and (40) we have

$$
\Psi(x, y)=B_{1}(\theta) e^{m \xi}+B_{2}(\theta) e^{-m \xi}-\frac{4 V}{m^{2}} \cot 2 \theta
$$

Again using (46) and (21), we have the stream function as

$$
\begin{aligned}
\psi(x, y) & =B_{1}(\theta) e^{m \xi}+B_{2}(\theta) e^{-m \xi}-\frac{4 V}{m^{2}} \cot 2 \theta \\
& +U(x+y)+V\left(x^{2} \cot \theta-y^{2} \tan \theta\right)
\end{aligned}
$$

Now on solving equation (43) we have

$$
\begin{aligned}
S & =\frac{1}{m^{2}}\left(B_{3}(\theta) e^{m \xi}+B_{4}(\theta) e^{-m \xi}\right) \xi+B_{7}(\theta) e^{m \xi}+B_{8}(\theta) e^{-m \xi} \\
& +B_{9}(\theta) \xi+B_{10}(\theta)
\end{aligned}
$$

where $B_{7}(\theta)=\left(\frac{B_{5}(\theta)}{m^{2}}-2 \frac{B_{3}(\theta)}{m^{3}}\right), B_{8}(\theta)=\left(\frac{B_{6}(\theta)}{m^{2}}+2 \frac{B_{4}(\theta)}{m^{3}}\right), B_{9}(\theta)$ and $B_{10}(\theta)$ are arbitrary constants. Again using (17), (21), (28) and (35) we have

$$
H_{\xi \xi}=\left(B_{11}(\theta)+B_{0} \xi\right) H_{\xi}
$$

where $B_{11}(\theta)=R_{\sigma}(\cos \theta-\sin \theta) U$ and $B_{0}=-2 R_{\sigma}$.

Now solving equation (49) we have

$$
H=B_{12}(\theta) \int e^{\left(B_{11}(\theta)+B_{0} \xi\right)^{2}} d \xi+B_{13}(\theta)
$$

where $B_{12}(\theta)$ and $B_{13}(\theta)$ are arbitrary constants depending on parameter $\theta$.

\section{Result and Discussion}

Stream lines patterns have been obtained for two different cases $k=-n^{2}$ and $k=m^{2}$. In the analysis we observe that when quadratic perturbation term, $A x^{2}+B y^{2}$ dominates over uniform perturbation term $U(x+y)$ then stream lines are hyperbolic in nature and there exist stagnation point, Figure 1 and 2. If uniform perturbation term $U(x+y)$ dominates over quadratic term $\left(A x^{2}+B y^{2}\right)$ then stream lines obtained are of wavy nature including some closed graphs, Figure 1(a), $(b),(c),(d),(e)$ and $(f)$.

Moreover for the solution in the case $(I I)$ when $k=m^{2}$ where the exponential terms dominates over all other perturbed terms resulting no wavy solution and hence there are stream lines patterns of hyperbolic nature including some closed curves shown in Figure $2(g),(h),(i),(j)$ and $(k)$.

At last we have shown some viscosity variation in three dimensional surface graphs shown in Figure $3(a),(b),(c),(d)$ which shows there are significant variation of viscosity in the flow field corresponding to the considered kinematical condition for the vorticity.

\section{Concluding Remark}

In this paper we have taken the vorticity distribution proportional to the stream function perturbed by the more general quadratic stream and obtained the exact solution of equations of motion of a finitely conducting incompressible fluid of variable viscosity under the presence of transverse magnetic field. Transformation of variables has been used to find the solutions in terms of one dimensional variable, depending on the single parameter $\theta$. Moreover, by means of graphs, we have presented stream line patterns and variation of viscosity corresponding to the various solutions. Further, we can get more exact solutions by taking different values of parameter $\theta, U$ and $V$. Moreover the result in all cases for $A=B=0$, recovers the result of Jamil and Khan (2006). This guarantees the correctness of the mathematical calculations.

\section{References}

Ames, W.F. (1965). Non-linear partial Differential equations in Engineering. New York: Academic Press.

Boisvert R.E., Ames, W.F. and Srivastava, U.N. (1983). Group properties and new solutions of Navier-Stokes equations. Journal of Engineering Mathematics, 17, p. 203-221.

Chandna, O.P. and Oku-Ukpong, E.O. (1994). Flows for Chosen vorticity function-Exact solutions of the Navier-Stokes Equations. Int. J. Math. and Math. Sci., 17(1), p. 155-164.

Chandna, O.P., Barron R.M. and Chew K.T. (1982). Hodograph transformations and solutions in variably inclined MHD plane flows. J Engg Math, 16, p. 223-243. 
Hayat, T., Naeem, I., Ayub, M., Siddiqui, A.M., Asghar, S., Khalique, C.M. (1988). Exact solutions of second grade aligned MHD fluid with prescribed vorticity. Nonlinear Analysis: Real world Applications, 46, p. 89-97.

Jamil, M. and Khan, N.A. (2006). Some exact solutions of equations of motion of a finitely conducting incompressible fluid of variable viscosity in the presence of transverse magnetic field by transformation method. ARPN Journal of Engineering and Applied Sciences, 1(2).

Kannan, K. and Rajagopal, K.R. (2008). Flow through porous media due to high pressure gradients. Applied Mathematics and Computation, 199, p. 748-759.

Martin, M.H. (1971). The flow of viscous fluid. Arch. Rat. Mech. Anal., 41(I).

Myers, T.G., Charpin, J.P.F and Tshehla, M.S. (2006). The flow of a variable viscosity fluid between parallel plates with shear heating. Applied Mathematical Modeling. 30, p. 799-815.

Naeem, R.K. (1984). Exact solutions of flow equations of an incompressible fluid of variable viscosity via one-parameter group. The Arabian Journal for Science and Engineering, 19(1), p. 111-114.

Naeem, R.K. and Jamil M. (2006). On plane steady flows of an incompressible fluid with variable viscosity. Int J of Appl Math and Mech., 2(3), p. 32-51.

Naeem, R.K. and Nadeem, S.A. (1996). Study of plane steady flows of an incompressible fluid of variable viscosity using Martins method. International Journal of Applied Mechanics and Engineering, 1(3), p 397-433.

Naeem, R.K. On exact solutions of Navier-Stokes equations for viscous compressible fluid. A majour paper of Master of science at University of Windsor, Ontario, Canada.

Naeem, R.K., Mansoor, A., Khan, W.A. and Aurangzaib. (2009). Exact Solutions of Steady Plane Flows of an Incompressible Fluid of Variable Viscosity Using $(\xi, \psi)$ - Or $(\eta, \psi)$-Coordinates. International Journal of Computational and Mathematical Sciences, 3, 1 .

Naeem, R.K.and Ali, S.A. (2001). A Class of exact solutions to equations governing the steady plane flows of an incompressible fluid of variable viscosity via von Mises variables. International Journal of Applied Mechanics and Engineering, 6(2), p. 395-436.

Nemenyi, P.F. (1951). Recent developments in inverse and semi-inverse methods in the mechanics of continua. Advances in Applied Mechanics. New York. 2.

Popovych, R. (1995). On Lie Reduction of the Navier-Stokes Equations. Nonlinear Mathematical Physics, 2(3), p. 301-311.

Siddiqui, A.M., Hayat, T., Siddiqui, J. and Asghar, S. (2008). Exact Solutions of Time-dependent Navier-Stokes Equations by Hodograph-Legendre transformation Method. Tamsui Oxford Journal of Mathematical Sciences. Aletheia University, 24(3), p. 257-268.

Sunil, Sharma, A. and Shandil, R.G. (2008). Effect of magnetic field dependent viscosity on ferroconvection in the presence of dust particles. J Appl Math Comput, 27, p. 7-22.
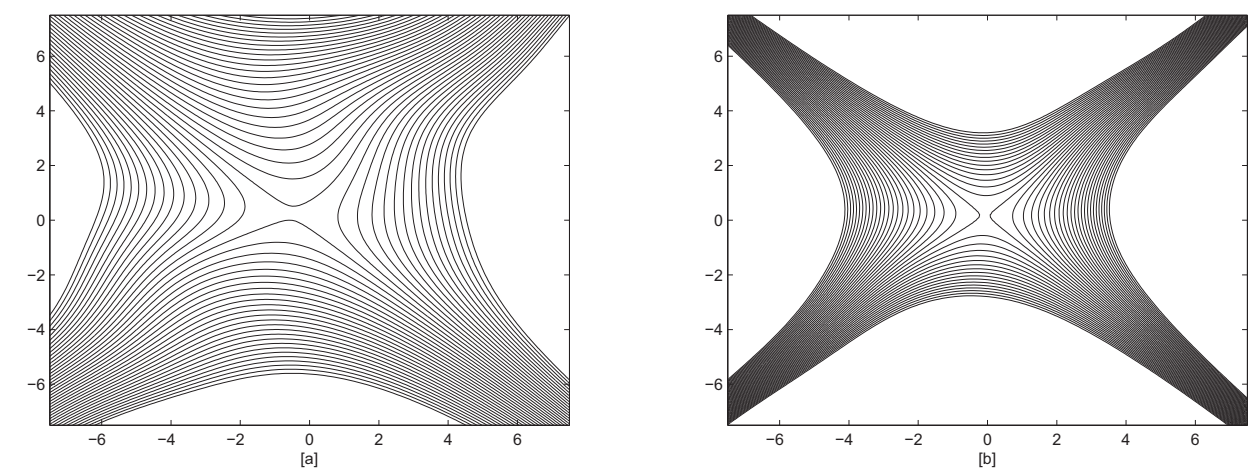

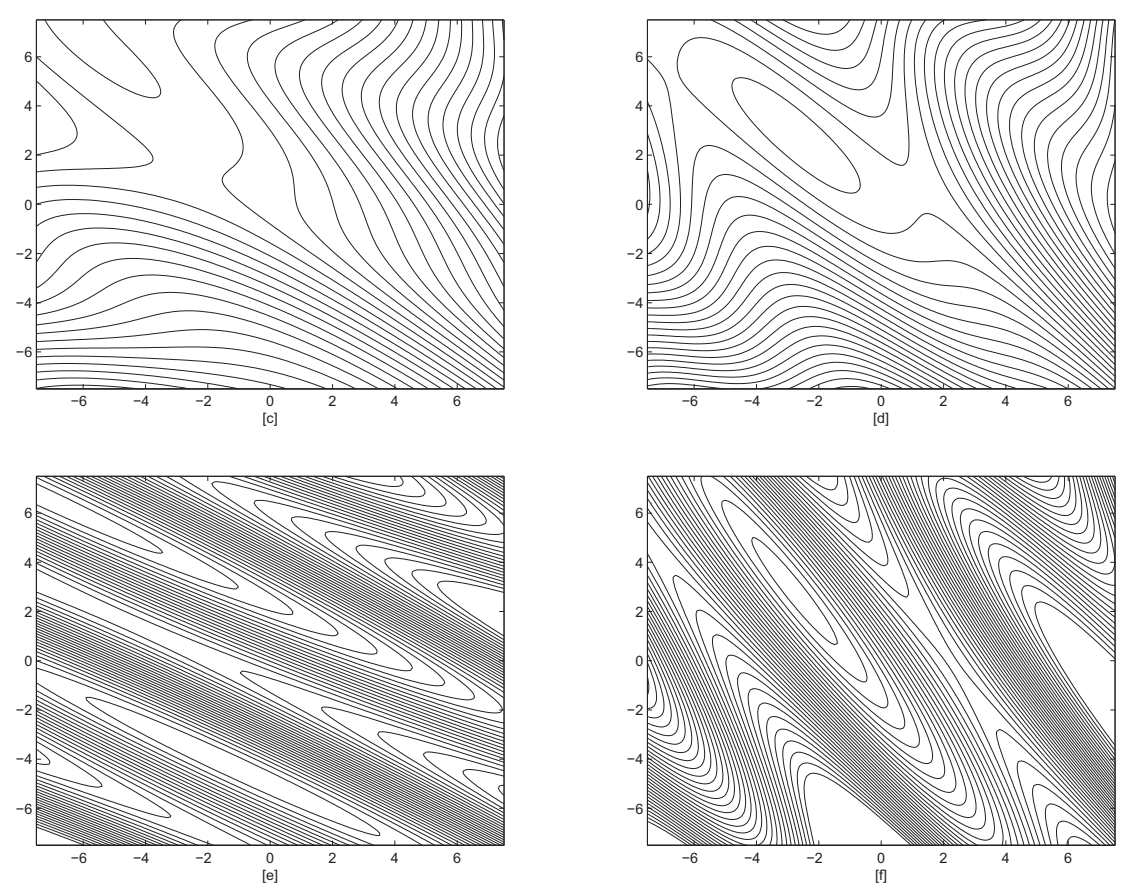

Figure 1.
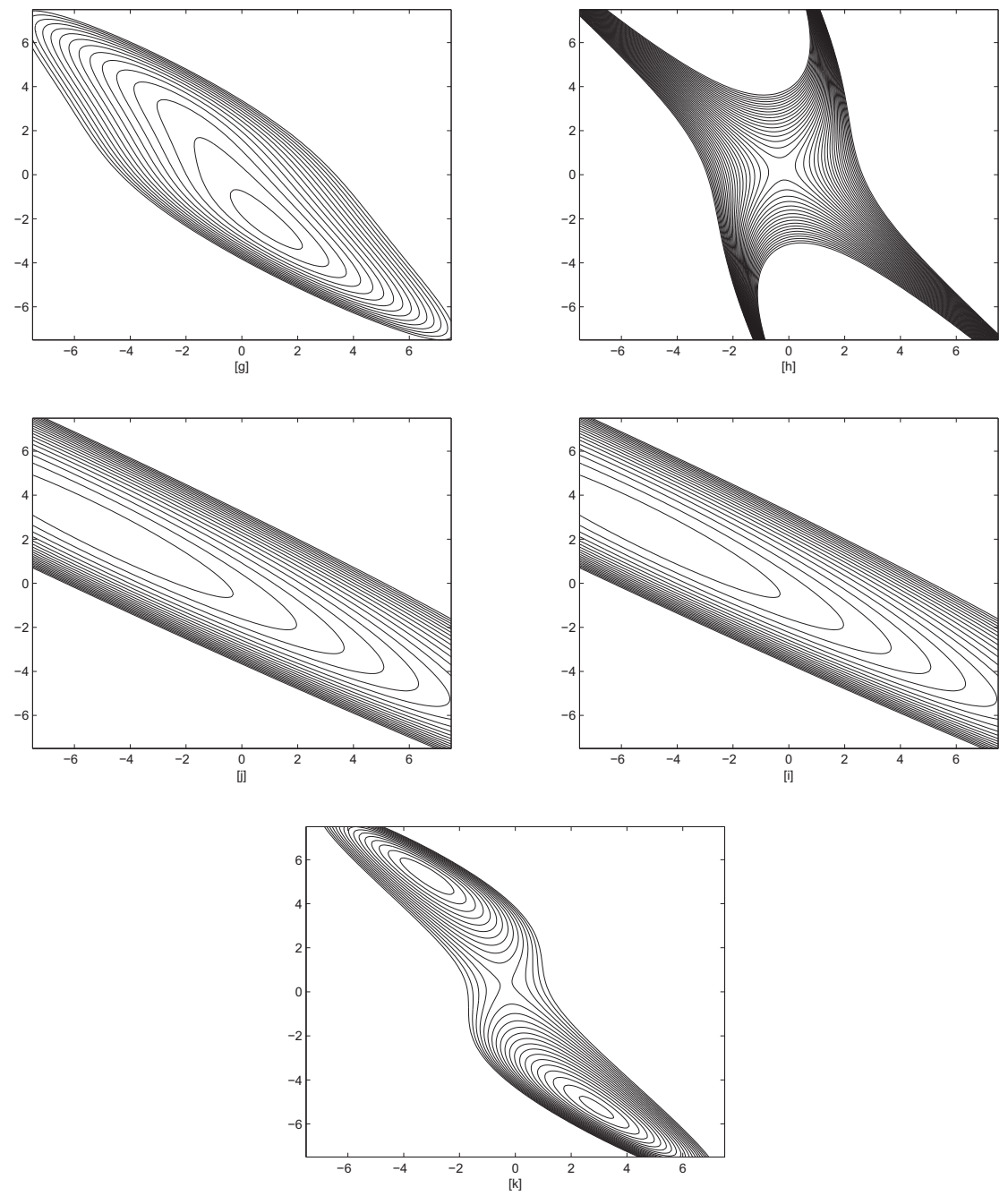

Figure 2. 

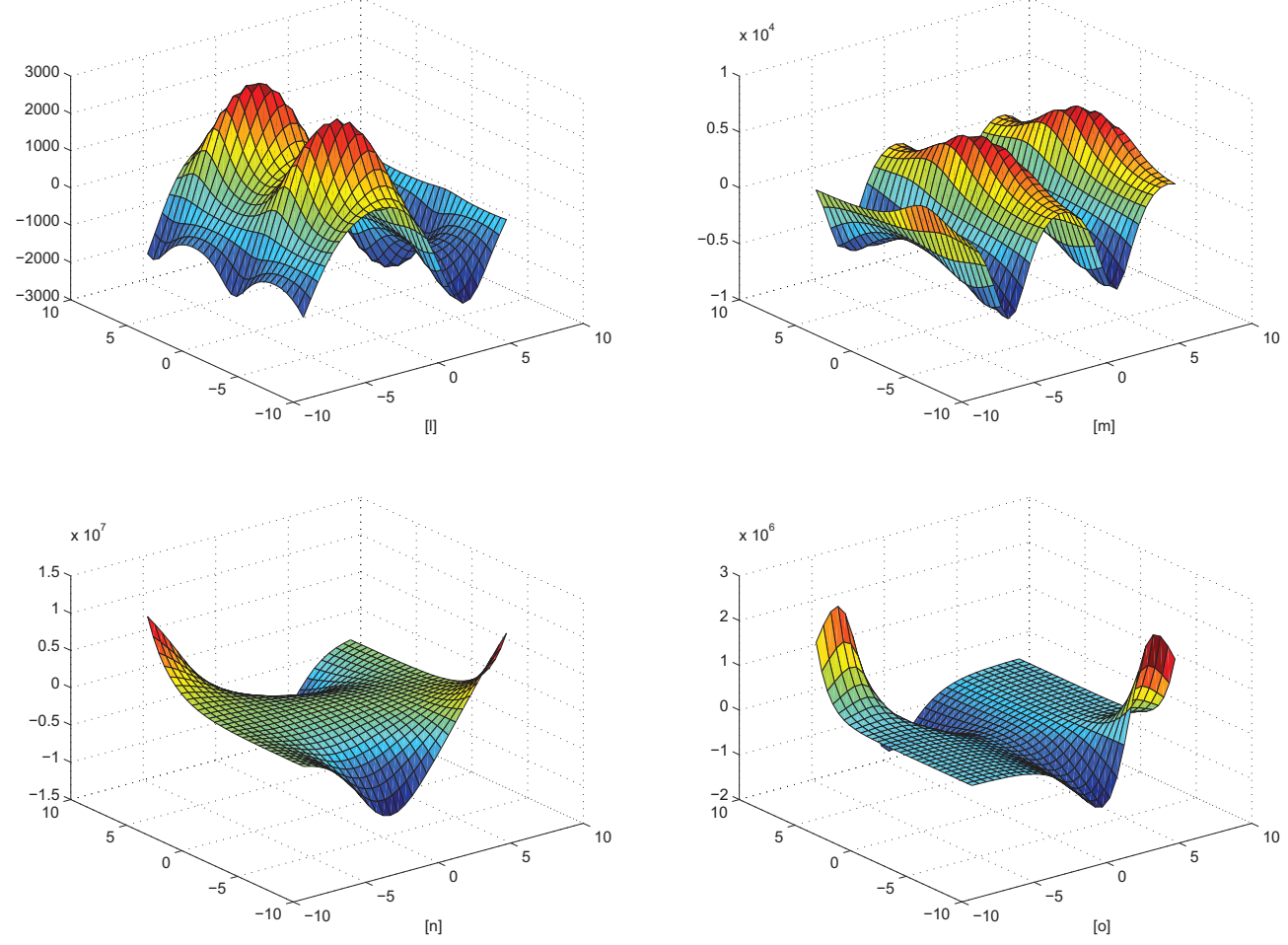

Figure 3. 